\title{
Article \\ Simulation-Based Public Transport Priority Tailored to Passenger Conflict Flows: A Case Study of the City of Zagreb
}

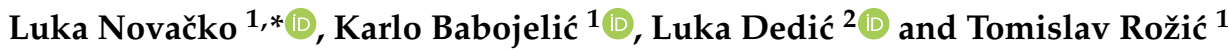 \\ 1 Faculty of Transport and Traffic Sciences, University of Zagreb, HR-10000 Zagreb, Croatia; \\ karlo.babojelic@fpz.unizg.hr (K.B.); tomislav.rozic@fpz.unizg.hr (T.R.) \\ 2 Prometis Ltd., HR-10000 Zagreb, Croatia; dedic.luka7@gmail.com \\ * Correspondence: luka.novacko@fpz.unizg.hr
}

Citation: Novačko, L.; Babojelić, K.; Dedić, L.; Rožić, T. Simulation-Based Public Transport Priority Tailored to Passenger Conflict Flows: A Case Study of the City of Zagreb. Appl. Sci. 2021, 11, 4820. https://doi.org/ $10.3390 /$ app 11114820

Academic Editor:

Roland Jachimowski

Received: 30 April 2021

Accepted: 21 May 2021

Published: 24 May 2021

Publisher's Note: MDPI stays neutral with regard to jurisdictional claims in published maps and institutional affiliations.

Copyright: (c) 2021 by the authors. Licensee MDPI, Basel, Switzerland. This article is an open access article distributed under the terms and conditions of the Creative Commons Attribution (CC BY) license (https:/ creativecommons.org/licenses/by/ $4.0 /)$

\begin{abstract}
Prioritizing public transport is one of the most effective measure to increase the attractiveness and competitiveness of public transport in relation to individual vehicles. The main goal of this study was to examine the possibilities for reducing the travel time of tram vehicles by giving priority at signalized intersections in terms of sharing the traffic lane with personal vehicles and under conditions of strong conflict flows with private transport. For this purpose, we used the simulation tool VISSIM and its module EPICS. A methodology for determining weighting factors for prioritizing public transport flows was developed based on conflicting passenger flows in public transport vehicles and passenger cars. Three scenarios were tested in the study area: (1) "do nothing"; (2) unconditional priority; and (3) conditional priority. The results showed that unconditional priority led to unsustainable traffic conditions for personal vehicles and public transport. In contrast, conditional priority reduced the travel time of public transport on certain corridors by between $7.64 \%$ and $18.76 \%$ in the morning peak period, and $5.60 \%$ to $22.50 \%$ in the afternoon peak period.
\end{abstract}

Keywords: public transport priority; VISSIM; EPICS; weighting factors; unconditional priority; conditional priority

\section{Introduction}

Traffic management in urban areas is focused on reducing the time losses of private and public transport vehicles caused by inadequate phase changing or phase duration at signalized intersections. To minimize time losses, traffic light management uses priority assignment techniques. The aim of this study was to examine in simulation tool the possibilities of reducing the travel time of tram vehicles by giving priority at signalized intersections under the conditions of sharing the traffic lane with personal vehicles and strong conflict flows with private transport. It also aimed to enable the running of private transportation by adjusting the weight factor priorities of public transport compared to the number of passengers in tram and cars during peak periods at intersections.

The aim of using the simulation tool was to test several variant solutions for prioritizing PT at intersections in the central part of the city in an economically acceptable and time-effective way. Direct application of PT priorities at intersections without prior simulation of different variant solutions could lead to heavy congestion, increased PT travel times and rejecting this type of traffic management. Direct implementation of traffic policy measures without prior testing in simulation models could lead to unnecessary additional costs for city authorities. PTV EPICS was chosen as a prioritization method because it uses weight factors to allow variable prioritizations of different traffic flows and vehicle types.

According to Sunkari et al. [1], priorities are divided into two groups: passive and active priorities. Passive priority techniques do not explicitly recognize the actual presence of public transport (PT) vehicles. Predetermined timing plans are used to provide some benefit to PT movements but do not require the presence of the PT vehicle to be active [1]. Active priority occurs when the detection of a PT causes the activation of a new signal 
timing pattern which overrides the existing pattern. In general, the improvement in active priority compared to the passive priority concept is that, in the former, priority is given only when the bus is present [1]. Passive priority methods are financially cheaper [2] and are conducted using cycle length adjustment, splitting phases, areawide timing plans and vehicle metering $[1,3,4]$. Passive priority, although beneficial for public transport (PT), through signal coordination that favors PT vehicles, can consequently have a negative impact on other modes of traffic and cause delays, additional stops and user frustration [5]. Sunari et al. in [1] divided active priority approaches into two strategies: unconditional (absolute) and conditional (relative) priority. Both strategies use four methods of active prioritization: phase extension, earlier start [6], special phase [6,7] and phase suppression [6]. Absolute priority strategies are manifested through unconditional vehicle passing and are intended to give priority to emergency services, whereas relative priority strategies are formalized by giving higher priority to PT vehicles $[1,3,4]$, while still considering other modes of transportation. Liu et al. in [8] noted potential shortcomings in applying active priority due to the required pedestrian and inter-green times at large intersections. Similar findings were made by Chandler and Hoel [9], who noted the negative effects of active priority on individual motorized traffic.

Modern methods use simulation models to reduce time and enable cost-effective and safe testing of the results of the proposed solutions. Simulation models have often been used as tools in applied research related to modeling, planning, development and optimization of traffic, traffic networks or traffic systems [10]. Therefore, testing the effects of prioritization in simulation tools has emerged as a standard in recent decades. Anderson and Daganzo [11] proposed the PT priority method using a mathematical model based on the Brownian motion concept. Using this concept, they developed an improved TSP (Transit Signal Priority) with a relative priority assigned to buses. The concept reduces the number of priority requests by about $50 \%$ and increases the reliability of the TSP system. In their research, Ghaffari et al. [12] proposed a methodology for addressing PT priority at the network level under variable demand conditions. Using this methodology, the system can recognize the temporal-spatial priority lane at times of reduced demand and mathematically formulate demand as a bi-level non-linear problem. The mathematical model was coded in MATLAB and combined with the VISUM simulation tool and showed the importance of considering hourly variations in demand when planning PT priority. Haitao et al. in [13] proposed methods for PT priority in bi-modal urban networks (urban networks with two intertwined modes of transport). Using mathematical models and the Three-Dimensional Macroscopic Fundamental Diagram (3D-MFD), the authors assigned weight factors to trams and cars based on the average vehicle occupancy. The results obtained from the macrosimulation suggest a possible increase in total passenger capacity through the application of different strategies depending on the situation. Ji et al. in [14] presented the results of an improved approach to tram priority assignment via an offline signal time scheduler and an online tram progression controller. The offline planner optimizes the progress of the tram through the network by synchronizing the green times depending on feedback about the progress of the tram while considering other vehicles. The results of the simulation, which was conducted in TransModeler, indicated the possibility of reducing the travel time of trams via recommended stop times at the station, recommended speeds while driving between stations, and extension of green times in the priority phases. Zhou et al. in [15] presented the results of research based on a proposed mathematical model that aimed to improve the functioning of the entire system by considering tram and car traffic. Through the goal function of minimizing the delay time for both types of traffic, and assigning higher weight factors for tram traffic, a balance between the total delay time in both observed traffic modes was achieved.

De Keyser et al. in [16] presented a comparison of the simulation tools used in PT prioritization. Scenarios from various countries (Croatia, Belgium, USA, Poland, Sweden, China, Egypt, etc.) covered various areas including signalized intersections and corridors to the entire network. Of the seven comparable studies, five used the VISSIM microsimulation 
tool [16-20], one study used ExtendSim [21] and one used FLEXSYT [22]. VISSIM is widely applied to the testing of assigned PT priority methods due to the variety of features it offers. In addition to the possibility of assigning priorities using a built-in algorithm module (the VisVap module built into the VISSIM program interface), it also provides the ability to connect to user-designed priority algorithms. Ou et al. in [23] presented VISSIM simulation model results in which the optimization method of prioritization was integrated using the COM interface. The optimization method was based on the spatiotemporal model of complex switch area operations. Conflicts of all tram lines and conflicts of tram lines with motorized traffic were considered in the presented mathematical method. The simulation results indicated a $26.6 \%$ reduction in delay time compared to the non-priority scenario and a $13 \%$ reduction in the delay time if the first-come-first-served (FCFS) priority strategy was considered. Stevanovic et al. in [24] presented the possibility of optimizing signal plans and TSP by integrating a genetic algorithm into the VISSIM evaluation environment. The results of the simulation of 12 signalized intersections indicated a reduction in the delay time for individual vehicles and PT compared to the simulations of the initial state.

In addition to research based on simulations, in many cities, after obtaining simulated results, researchers have also implemented the methods of PT prioritization at real city intersections and corridors. The implementation of relative priority based on simulated data was carried out in Krakow, Poland, on four corridors with a total length of $19 \mathrm{~km}$ and 30 intersections [25]. The results on the usage of an adaptive traffic management module called BALANCE [26] in combination with a module for public transport priority of PT called EPICS [27] indicated the possibility of reductions in PT travel time by an average of $10 \%$, which caused an increase in motorized traffic by an average of $9 \%$ in the morning peak period but a decrease of $10 \%$ in the afternoon peak period. In addition to Krakow, successful implementation has been carried out in three other cities in Poland-Gdansk, Gdynia and Sopot-at a total of 150 intersections. According to [28], the implementation resulted in an overall reduction in travel time of private vehicles by $18 \%$ and PT by $9 \%$. Klanac et al. in [29] presented a comparison of local and coordinated management via EPICS with the actuated-coordinated Ring Control Barrier (RBC). The results of local management without coordination indicated traffic time reductions of more than $10 \%$, whereas comparison of the results with coordinated management indicated a reduction of up to $5 \%$, confirming better efficiency of EPICS when using it for local optimization.

The above literature review shows that the usage of microsimulation tools to test proposed traffic solutions is common in related research. Even though various micro and macrosimulation tools are used to evaluate the proposed optimization and prioritization solutions, VISSIM is notable among the many simulation tools. Many studies refer to the improvement of existing priorities resulting from areawide timing plans by designing signal offsets in a coordinated signal system using PT travel times. These methods are limited to lengthening or shortening phases depending on the announcement of priority vehicles within the time frame provided by the PT timetable. Some of the studies considered only the results of PT improvement, whereas others considered prioritized and non-prioritized traffic. According to case studies mentioned in the literature review, using the network-level optimization module (BALANCE) and the local intersection-level prioritization module (EPICS) it is possible to achieve optimal solutions and reduce the average waiting time by giving priority to PT without major impacts on individual motorized traffic. Even though a study presented in the literature review showed that EPICS has a greater impact on local optimization than on coordinated optimization, this cannot be confirmed due to the lack of available literature. To our knowledge, with the exception of the cases presented in the literature, EPICS has not been used elsewhere and the results presented in this article are based on one of the largest microsimulation models, giving priority to trams, to date. Based on data collected in the City of Zagreb, Croatia, from a traffic network of 49 intersections, a VISSIM microsimulation model was designed. Using EPICS for the priority model and a mathematical model to determine the PT weight factors, the conditional PT priority was conducted for 29 simulated intersections. 


\section{Methodology}

\subsection{Study Area}

The analyzed area represents the transport network and public transport areas in the central part of the City of Zagreb, Croatia (Figure 1). A total of 14 of the 15 tram lines pass through this area, and largely share the road with private motorized transport. The study area has 49 signalized intersections and pedestrian crossings, and trams pass through 29 signalized intersections. Passenger transport by trams largely takes place on streets on which the network of tram lines coexists with road motor traffic. Thus, over time, the traffic volume has increased in limited spatial areas.



Figure 1. Study area in the center of City of Zagreb.

About $40 \%$ of tram lines in the City of Zagreb are not physically separated from individual traffic, so the speed and accuracy of the given timetable of tram lines largely depend on the density of individual traffic. The fact that trams do not have an announcement or a priority of passing through signalized intersections is also important for the low speed and regularity of tram traffic.

There have been no significant conceptual changes in public urban passenger transport for many years, with the exception that, during the past ten years, the tram and bus fleet has been largely renewed with modern low-floor vehicles. In the current traffic situation, the regulation of conflict points on the main tram corridors is achieved by a traffic light system that does not recognize the priority of tram traffic. As a result, the tram speed on some tram sections is reduced to such an extent that the quality of tram traffic is significantly impacted, thus affecting the choice of this form of transport for the daily commute of citizens (Figure 2). 


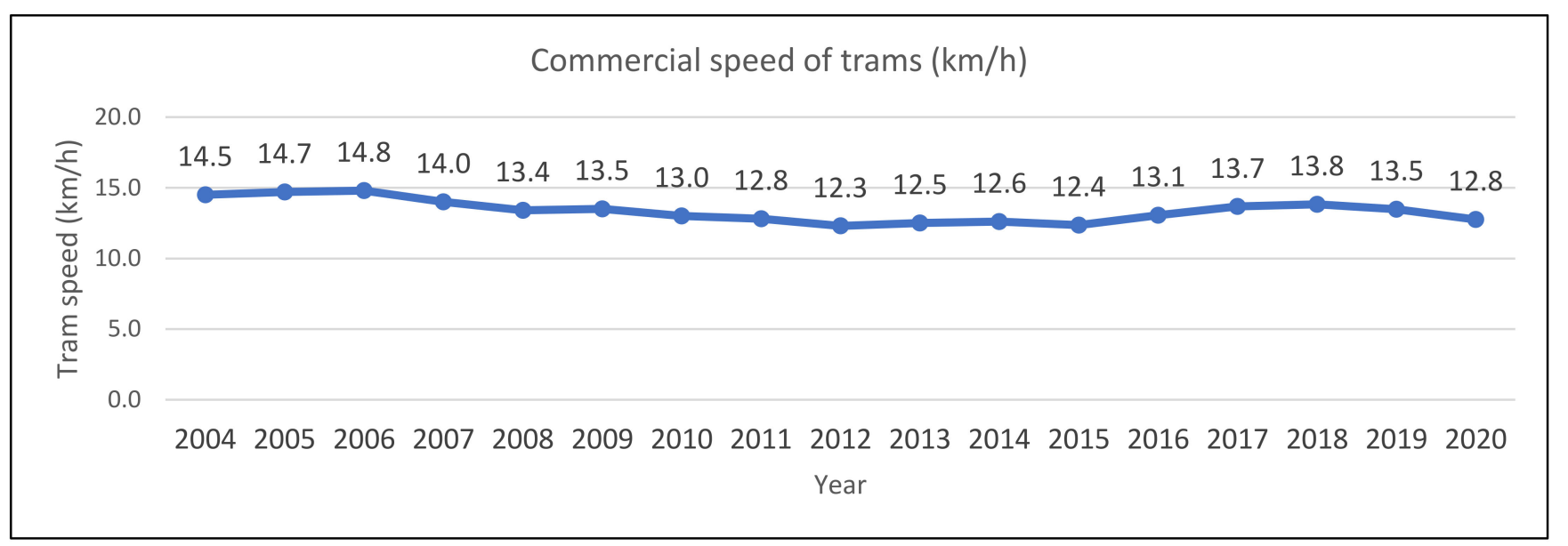

Figure 2. Commercial speed of tram lines in City of Zagreb [30].

A system of special lanes (not physically separated from private vehicles) to achieve the priorities of public urban passenger transport is not fully functioning because traffic police have not been able to implement it efficiently. As a result, in general, the expected effects of this measure to improve public transport have not materialized.

\subsection{Data Collection}

Three types of counting were performed during September and October in 2019 in the analyzed area: (1) counting of vehicles at intersections; (2) counting of passengers in trams; and (3) counting of passengers in private vehicles. This provided a complete (objective) picture of the relationship of traffic flows, both in terms of their number (vehicles in space and time) and quantity, i.e., the number of passengers.

Traffic counting was performed at all approaches from 49 signalized intersections. Regardless of the timetables, the tram trains were counted. This approach was necessary because systematic traffic counts are not conducted in Zagreb, nor does the existing installed equipment enable vehicle counting and classification.

An objective picture regarding the justification of the implementation, and the necessary level, of public transport priorities in the central part of the city best describes the relations between passenger flows in public and individual transport. As automatic passenger traffic counting is still not performed in public transport vehicles, for the purposes of this research, three goals of the tram passenger counting methodology were set: (1) the most accurate assessment of passengers on tram lines should be achieved; (2) the counting process should have a minimal impact on passenger behavior and comfort; and (3) counting should be cost-effective.

The counting of passengers in public transport vehicles was carried out simultaneously with the counting of passengers in individual transport. The counting of passengers in personal vehicles was undertaken by counters in a stationary position at the approaches; using a quick visual inspection of the sampled number of vehicles, the average number of passengers in the vehicle was able to be determined.

The aim of counting passengers in public transport was to obtain basic parameters that characterize the movement of passengers on each route of the network, for all lines, such as: entrances and exits of passengers along the line, number of passengers between two stops, passenger flow, the mean length of travel and boarding and alighting of passengers at stops. For the purposes of this research, the counting of public transport passengers was performed by pre-trained counters that counted the passengers in the trams. 


\subsection{Public Transport Priority}

The microsimulation model of the current and future state was created using the VISSIM software tool, and the simulation of public transport vehicle priorities was performed using the EPICS software module, as part of VISSIM.

To address the problem of increasing transport demand at signalized intersections, an increasing number of intersections are subject to traffic-dependent signal plan management. The traditional variant of these systems is based on the principle of impulse (detector) reaction, i.e., control algorithms consist of highly complex elements (if-then queries), especially regarding prioritizing public transport vehicles. A more modern approach to adaptive optimization, such as EPICS, is based on an iterative approach. EPICS was first intended to replace existing priority measures of public transport vehicles [31]. Initially, it focused only on public transport vehicles, but with further developments it became a control method providing real-time optimization of traffic control parameters for all users [29]. EPICS allows special attention to be paid to the time of arrival of public transport vehicles from the moment of the first announcement on the sensor (check-in detector) to the passage of the stop line (check-out detector). Unlike private transport flows, PT vehicles usually appear discretely at intersections. Therefore, due to the desired possibility of prioritization, the vehicles must be considered as single events. Differences in the arrival time of vehicles that occur due to occasional disturbances are respected by considering them to have a trapezoidal probability distribution, rather than treating them as a point (Figure 3) [27]. To obtain the probability density function, it was necessary to determine the ranges of PT vehicle time arrivals to the stop line for each intersection. That was accomplished by measuring arrivals in the field and entering the time values P1, P2, P3 and P4 in the EPICS program, where P1 represents fastest, P2 typical, P3 typical slow and $\mathrm{P} 4$ slowest travel times to the stop line.

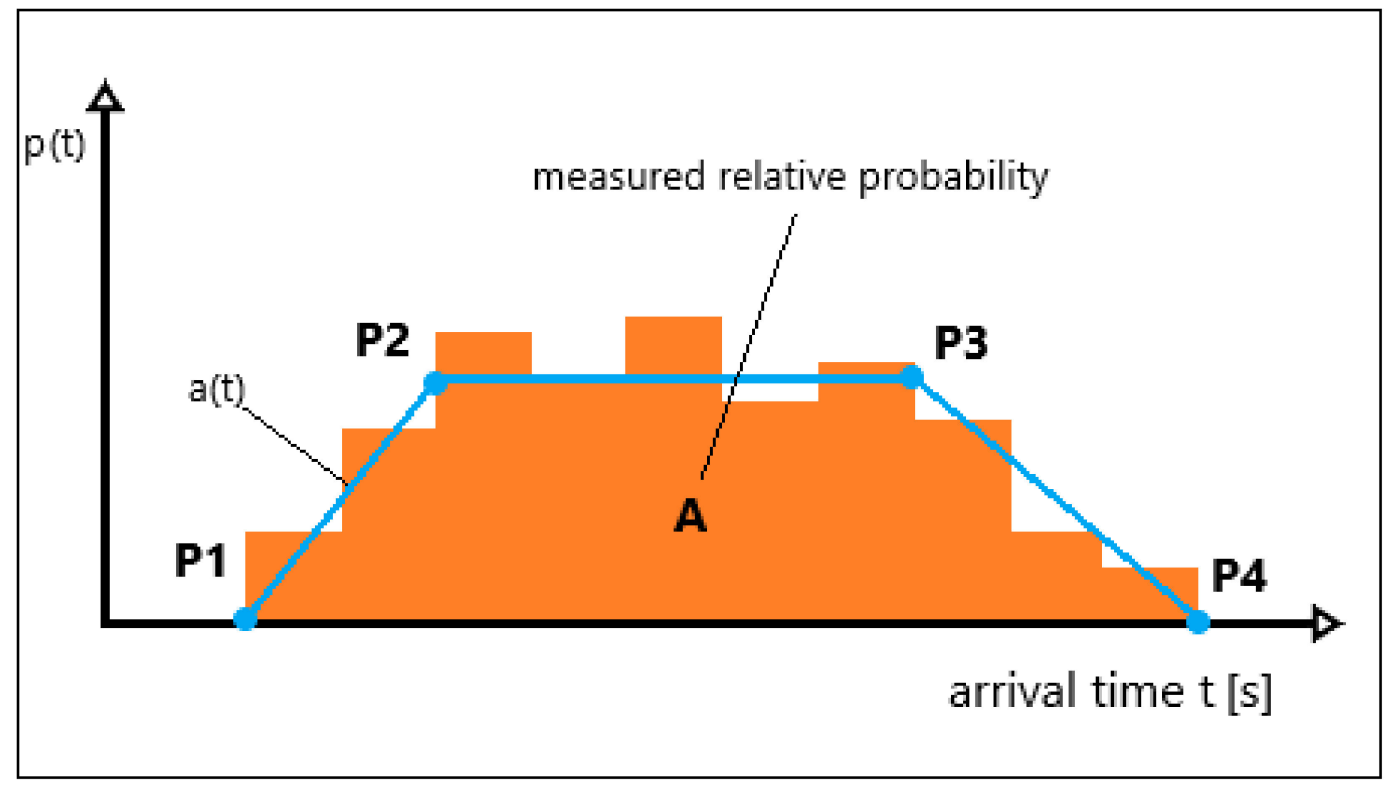

Figure 3. Trapezoidal probability density function of the travel time of a public transport vehicle from the first announcement to the stop line [27].

If the public transport vehicle uses a common lane with private transport, EPICS gives priority to the PT vehicle by extending the duration of the green light or switching on the signal group to clear the queue and allow unobstructed passage of the public transport vehicle. The relevant quantities entering the target function of the optimization of EPICS are the total delay and the number of stops of vehicles, which are calculated by summing up all detected traffic flows, each with a configurable weight. Additionally, there is the possibility to compare with a reference signal plan to guarantee coordination of the local 
control with the surrounding network as good as possible. It can be provided offline by a traffic engineer or generated online by means of a suitable network control such as BALANCE [27].

Based on the input data, the efficiency models calculate different variant process optimization scenarios using the Performance Index (PI) shown in Formula (1) [27].

$$
P I(s p)=\sum_{s g \in S G} \alpha_{s g} D_{s g}(s p)+\beta \Delta(r e f, s p)
$$

where:

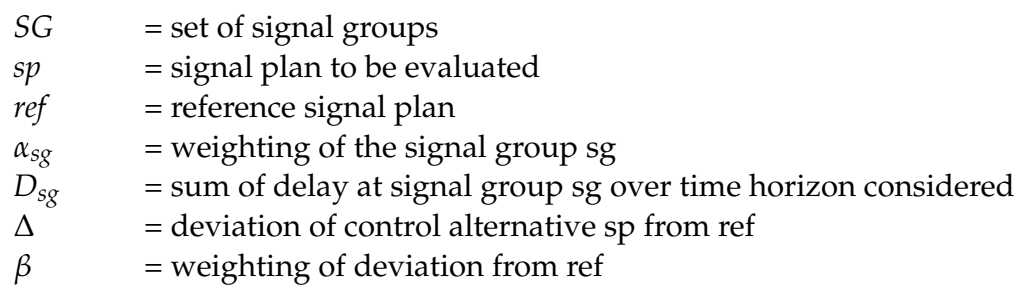

From this formula, the waiting times of each vehicle are calculated depending on the management scenario, and the scenario with the lowest total waiting time of all vehicles is selected as the optimal one. The only element in the EPICS module that can influence the priority of individual signal groups is the weight factor $\alpha_{\text {sg }}$. In [27], it is stated that a value between 50 and 100 is recommended in the case of PT prioritization. By assigning a higher value of the weight factor $\alpha_{\text {sg }}$ to the signal group in which the public transport is defined, the model strives to reduce the delay time of that signal group to achieve the minimum PI factor. The method of determining the weight factor $\alpha_{\mathrm{sg}}$ is explained in Section 3.1. If BALANCE is not used for network coordination, the second part of Formula 1. can be excluded.

An attempt to minimize PI is made through the control model, which is an integral part of EPICS. EPICS is stage and interstage based. The control model calculates, for the given time horizon $\mathrm{T}$, a stage sequence that minimizes the PI. The signal plan with the lowest PI is accepted and transferred to the traffic light control. The operation of the mentioned optimization is performed in two steps. In the first step, the time horizon is split into a grid with a width of $5 \mathrm{~s}$. Here, the stage sequence in principle is set. In the second step the fine tuning takes place, i.e., the starting times of the inter-stages are optimized with one second precision. The first step uses a (slightly modified) branch-and-bound algorithm and the second one an ordinary hill-climbing algorithm [27].

\subsection{Comparison of Public and Individual Transport}

One of the key problems of traffic flows in the central part of Zagreb is the inseparability of tram and individual traffic. The use of dedicated PT lanes by car drivers is common, and the coordination of traffic light devices is mostly adapted to individual rather than public transport. This results in a constant decline in the competitiveness of public transport. Even though private transportation is favored at signalized intersections, the modal split in favor of cars exceeds the existing capacity of signalized intersections. Due to the excess transport demand of private transport, a level of service $F$ was determined at $75 \%$ of the analyzed intersections in the peak periods. The clear solution is to change the transport policy in favor of public transport and increase its competitiveness using various measures, with special emphasis on the application of priorities for trams at intersections. In addition to the general assessment of traffic in the central area of the City of Zagreb, it is necessary to determine the relationship between individual and public transport in terms of the number of transported passengers.

Even though it is of key importance for the implementation of a certain transport policy, this aspect has not generally been considered in the traffic studies and research conducted to date in the City of Zagreb. As the function of the entire transport system is to meet the needs of people who use different transport modes, traffic planning and 
modeling should, at its core, address passengers rather than vehicles. To assess the current relationships between individual and public transport, additional research was conducted. In terms of individual and public transport, 29 key intersections in the central part of the City of Zagreb were analyzed in detail. The total car volume in two-hour peak intervals (morning and afternoon) is approximately 91,000 Passenger Car Units (PCUs). The total traffic volumes during the peak periods are approximately the same, i.e., the afternoon peak interval is slightly higher (by $0.5 \%$ ). Given the high levels of saturation and the dominant $\mathrm{F}$ level of services, prioritizing public transport at signalized intersections should be preceded by proposals for alternative management of individual traffic flows (rerouting), in addition to a campaign to encourage the use of public transport, for which the quality of service will be raised due to being given priority at intersections.

A comparison of individual and public transport in terms of the number of passengers was carried out at all intersections on the corridors where public and private transport intersect. If the total number of passengers recorded in the two peak periods (7-9 $\mathrm{h}$ and $15-17 \mathrm{~h}$ ) is considered, almost the same number of passengers use individual and public transport. Similarly, the difference in passenger numbers between the morning and afternoon peak periods is negligible. Figure 4 shows ratios of passenger flows in private vehicles and public transport on five most saturated intersections in study area.

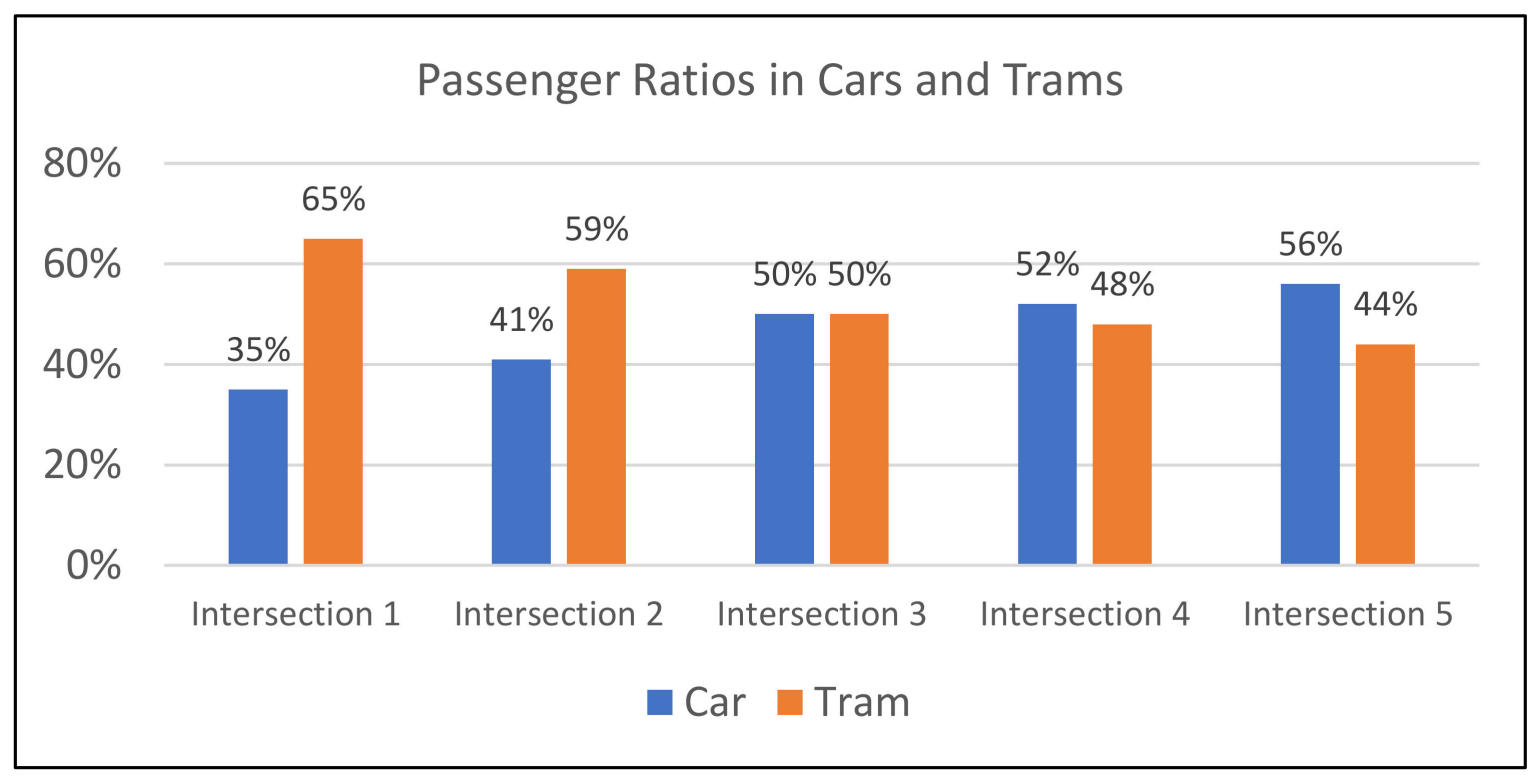

Figure 4. Passenger ratios in cars and trams on five most saturated intersections.

For the future strategy of traffic light system management in the center of the City of Zagreb, it is extremely important to determine the intensity of the proposed changes. Even though it is clear that public tram traffic should be given priority at intersections (which does not currently exist), the question about the extent to which this should be done so that individual traffic is not congested and causes additional delays in tram traffic.

An important guideline in determining this measure should be the number of flows of passengers in private and public transport intersecting at a particular intersection, rather than simply the number of approaching vehicles. As an example, the following Figure 5 shows the relationships of "conflicting" passenger flows in passenger cars (passenger/hour) and trams (passenger/hour) at the five most saturated intersections in the morning peak period from 7 AM to 9 AM. "Conflicting" flows represent flows of passengers in cars and trams that cannot pass through intersections at the same signal stage. The ratios of these flows show that there is a significantly higher number of passengers in public tram transport waiting for their green light than in passenger cars on the five most saturated intersections. 


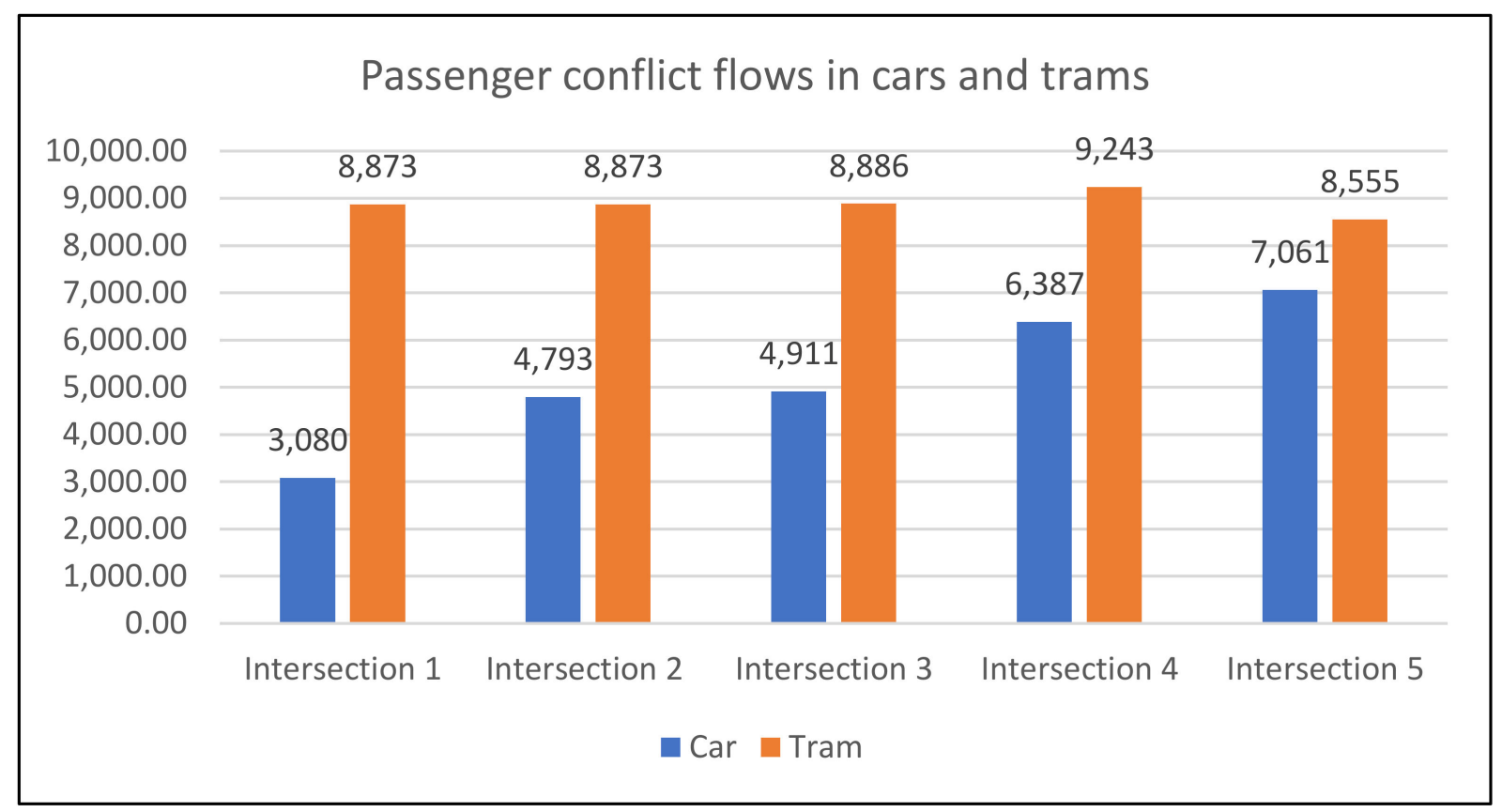

Figure 5. Passenger conflict flows in cars and trams on five most saturated intersections.

For traffic light control management in the central area of the City of Zagreb, the previously presented relationships will be one of the main guiding principles for measuring the level of priorities to be given to public transport in relation to the current situation of dominant individual transport. The aim is to determine the weight factors that determine the priority level of PT based on the relationship of conflicting flows of passengers in private transport and PT.

\section{Development of Microsimulation Model}

Microsimulation models are dynamic, stochastic, discrete modeling techniques that simulate the movement of individual vehicles based on car following, lane changing and gap acceptance algorithms that are updated several times each second [32]. Authors in [33] analyzed majority of existing car-following and lane changing models in simulation tools. These interactions between vehicles provide a basis for calculating vehicle delay times and other parameters important for evaluation. Their flexibility allows modeling of complex traffic operations. Due to several parameters that need to be adjusted to reflect the actual state of the study area more accurately, traffic modeling can be a long-term process. Figure 6 shows the traffic network of the study area in the microsimulation tool VISSIM.

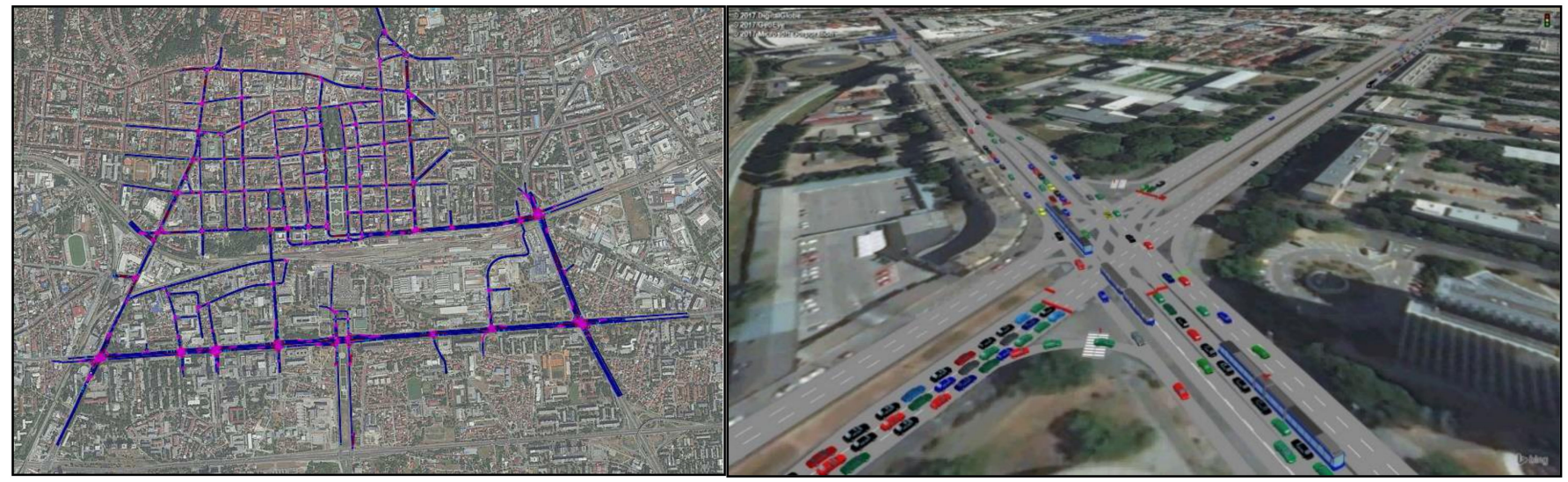

Figure 6. Microsimulation model of the study area in VISSIM. 
The developed microsimulation model is complex and consists of, among other components:

- 1127 links and connectors.

- 210 routes.

- 20 signal controllers with 49 signal plans.

- 163 nodes for evaluation.

Special attention is paid to the calibration of the model itself, and especially the driver's behavior when following and lane changing, to adapt the model as much as possible to local conditions.

\subsection{Determination of Weight Factors for PT Prioritization}

In EPICS, priority is represented by the weight factor $\alpha_{\mathrm{sg}}$ for the signal group that includes PT. The weight factor for PT prioritization is in the range of 50-100. A factor of 50 means that the priority of the PT is minimal, and 100 means that the priority of the PT is unconditional. By interpolation within the range of $50-100$, the weight factors for each signal group used by the PT can be determined. According to the methodology suggested in this research, the weighting factors should be determined on the basis of the relationship between the number of passengers in PT and the number of passengers in cars in conflicting traffic flows.

Based on the data obtained by counting at intersections and in PT, if the signal group with PT has T passengers, and the conflicting signal group with passenger cars has $\mathrm{C}$ passengers in the analyzed time period, then it is proposed to assign a weighting factor to the PT signal group according to the Formula (2):

$$
\alpha_{s g}=50+\left(\frac{T}{T+C}\right) * 50
$$

where:

$\alpha_{s g}$-weighting factor of signal group for PT priority;

$T$-number of PT passenger flows;

$C$-number of car passenger flows.

\subsection{Validation of Microsimulation Model}

Validation of the model was undertaken by comparing the output simulation results of the model with independent data that were observed during data collection and were not considered in the form of calibration [32]. Validation is an assessment of the quality of calibration and thus ensures the reliability and accuracy of the prediction of the traffic model. The validation of the simulation model was undertaken using the GEH statistic [34] using Equation (3):

$$
G E H=\sqrt{\frac{2 *(M-C)^{2}}{M+C}}
$$

where:

$M=$ traffic volume from model;

$C=$ real-world traffic count.

Regarding traffic modeling of the initial state, when comparing traffic volumes, a GEH coefficient of less than 5 indicates a good validation of the simulation model. Furthermore, $85 \%$ of the total traffic volume should have a GEH coefficient of less than 5 . Conversely, if the coefficient is greater than 10, the results of the comparison indicate a lack in the models ability to represent the actual situation [34].

According to [34], it is necessary to place the generated travel times within a deviation of $15 \%$ to $85 \%$ of travel routes to compare the accuracy of travel times. These data, which were collected by field testing, must also be correlated with the actual time (peak hours, day of the week) being modeled. The accuracy of the travel time is greater for the validation of public transport vehicles due to their predetermined routes and the smaller amount of 
data for comparison. Pedestrian flows can significantly affect the movement of traffic. The impact of pedestrian flows is especially pronounced at right turns at signalized intersections and significantly affects the reduction in the level of service.

The validation of individual flows was assessed using the GEH statistic described earlier. In the morning peak period, the number of traffic intersection approaches with a GEH coefficient of less than 5 was $59 / 65$, equal to $90.7 \%$, whereas in the afternoon peak period, the number of intersections with a coefficient of less than 5 was $57 / 65$, i.e., $87.7 \%$ (Table 1).

Table 1. Validation of microsimulation model using GEH statistics.

\begin{tabular}{ccc}
\hline & $\begin{array}{c}\text { Intersection Approaches } \\
\text { with GEH <5 }\end{array}$ & Percentage [\%] \\
\hline Morning peak period & $59 / 65$ & 90.7 \\
Afternoon peak period & $57 / 65$ & 87.7 \\
\hline
\end{tabular}

Validation of PT in the model was performed by comparing the travel times of trams that run along the entire observed corridor. The travel time must not deviate by more than $15 \%$ from the actual measured time. In the morning peak period, deviations for individual lines between the measured and modeled values ranged from $5.89 \%$ to $15.38 \%$, whereas in the afternoon peak period, these values ranged from $3.33 \%$ to $13.3 \%$. Figure 7 shows the validation of travel time of the longest tram line in study area.

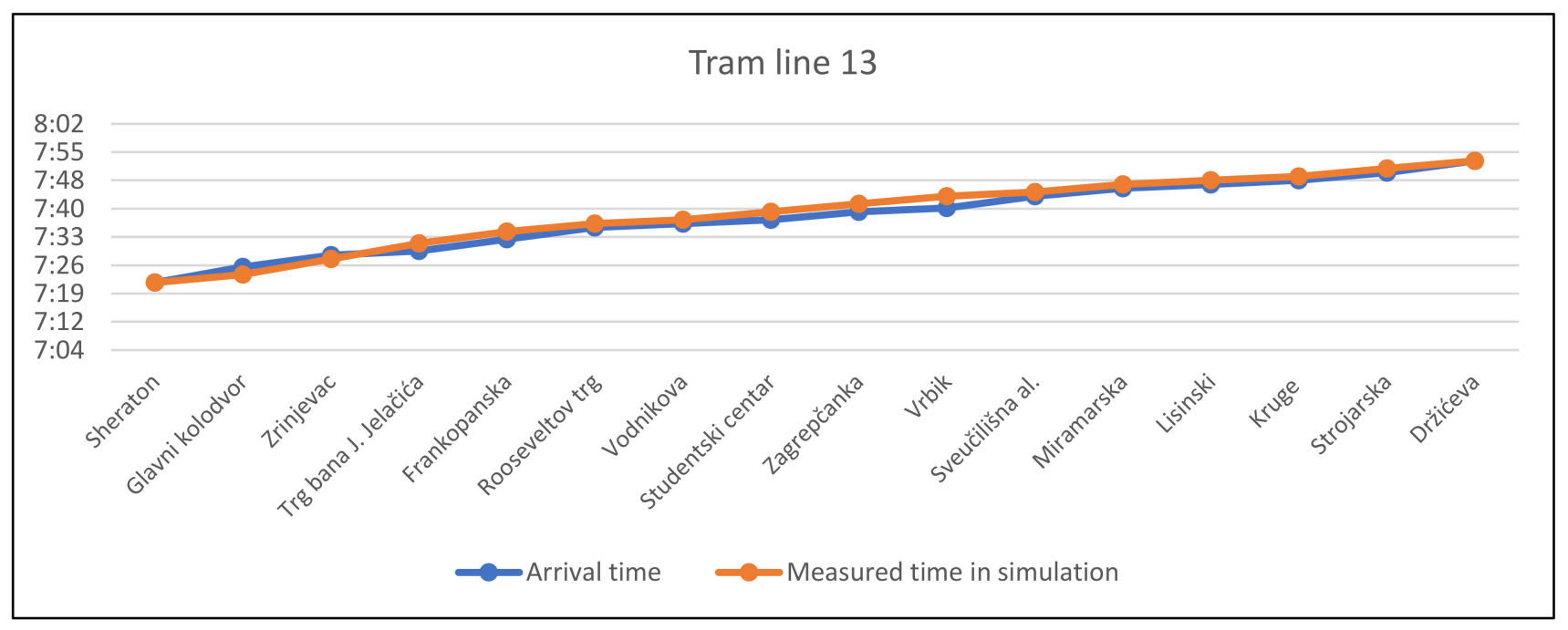

Figure 7. Comparison of arrival time and measured time in simulation of tram line 13.

\section{Evaluation of Results}

Based on the traffic flow trends and traffic forecast data taken from [35], it can be estimated that, with stable economic growth, moderate growth in traffic demand, of $1.5 \%$ on average per year, will occur over the coming 5 years; the overall five-year increase during this period will be $7.7 \%$. The impact of the COVID-19 pandemic has not been considered in the forecasts, because data on traffic volume trends in 2020 are not yet known; although working from home encourages less use of cars, due to the fear of infection there is less use of public transport.

Three scenarios were analyzed through 20 calibrated simulation model runs for each scenario assuming moderate 5 -year traffic growth of $7.7 \%$ :
1. "do nothing".
2. unconditional PT priority.
3. conditional PT priority. 


\subsection{Do Nothing}

The general increase in individual traffic of $7.7 \%$ compared to the current situation will lead to congestion of the considered transport network. At the quantitative level, the results within the individual transport system are expected to be significantly worse. Total maximum queue lengths are $12.45 \%$ higher and total waiting time is $19.39 \%$ higher (Figure 8 ).

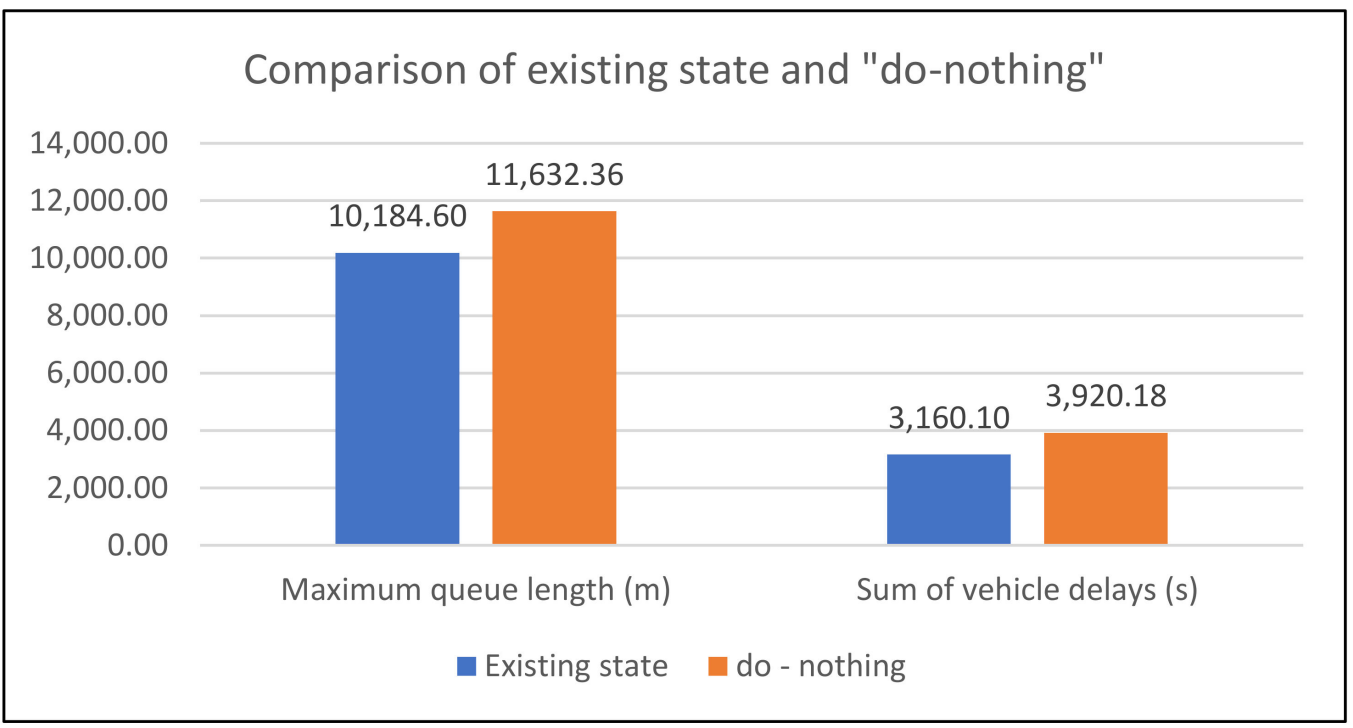

Figure 8. Comparison of maximum queue length and sum of vehicle delays of existing state and scenario "do-nothing" in the morning peak period.

In the corridor of study area in which 14 tram lines are located, and trams are not separated from individual motor traffic, the average travel time of tram vehicles in the north direction increases by $4.03 \%$, and in the south direction $8 \%$.

From the Figure 8, it can be concluded that the study area cannot accept increased traffic demand without specific organizational, regulatory and/or infrastructure measures.

\subsection{Public Transport Priority}

Signal control management in public transport priority assumes the allocation of the green phase to public city transport vehicles when encountering traffic lights. Giving priority to public transport is a measure implemented to limit individual motor traffic as one of the most significant positive effects of sustainable transport development in cities, which is reflected in the reduction in greenhouse gases and other positive effects related to environmental protection and energy efficiency.

\subsubsection{Unconditional Priority}

The unconditional priority of trams, after only $30 \mathrm{~min}$ of simulation runs, leads to a blockade and halting of traffic flow in the analyzed corridor of the central part of the City of Zagreb. Due to the high frequency of tram vehicles along the corridor, frequent minimal green times on opposite approaches create large queues that lead to unsustainable traffic conditions and oversaturation of vehicles at neighboring intersections. In the morning peak period, as many as $44.34 \%$ of vehicles failed to enter the network. It can be noted that due to the blockade by individual motor vehicles of the corridor through which the largest number of tram lines pass, the average travel time of trams would increase by $13.03 \%$ compared to the existing fixed control of signal controllers (Figure 9). 


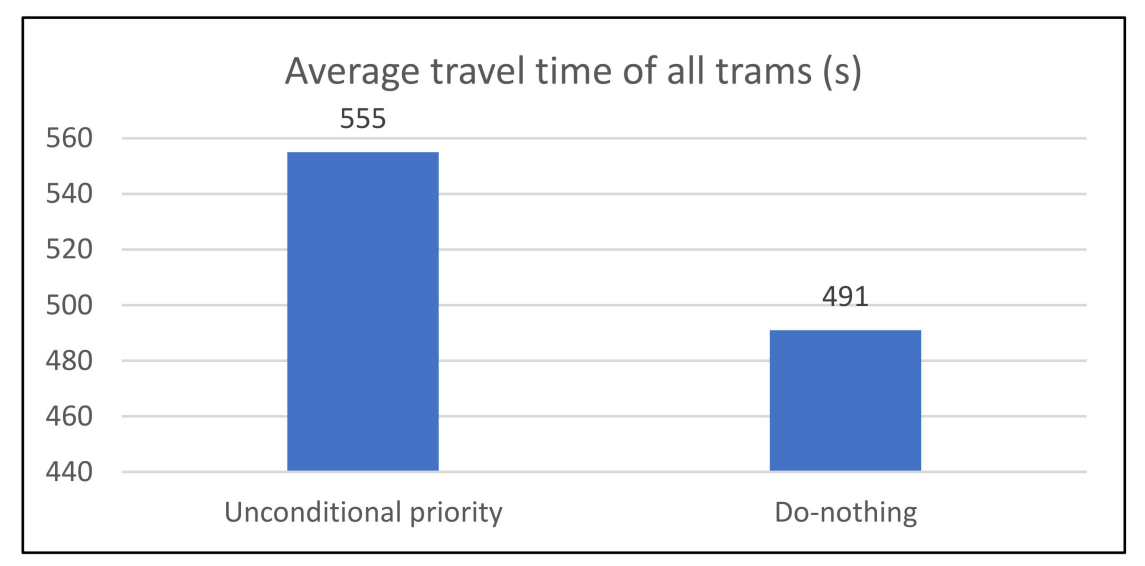

Figure 9. Comparison of average travel time of all trams (s) in scenario (2) and scenario (1).

Based on the conducted analysis using a simulation tool, it can be concluded that the introduction of unconditional priority of tram vehicles in the analyzed central part of the City of Zagreb would lead to complete traffic congestion in individual and tram traffic; thus, the analyzed scenario is not realistic.

\subsubsection{Conditional Priority}

To attempt to enable the functioning of the traffic system in the central part of the City of Zagreb without construction interventions and while maintaining a certain priority of tram lines, changes were introduced in relation to the previously analyzed unconditional priority. At intersections at which large queues were created during the unconditional priority simulation, the weight coefficients in the EPICS software tool were given conditional priority to tram traffic.

This type of priority of trams is called conditional because trams at these intersections are not given priority passage; rather, priority depends on the ratio of the number of passengers in individual vehicles and trams based on Equation (2).

The average reduction in travel time in the morning peak period varied from $7.64 \%$ to $18.76 \%$. In the afternoon peak period, improvements ranged from $5.60 \%$ to $22.50 \%$. It was noticeable that larger reductions in travel time were achieved in the corridors in which trams were separated from individual traffic.

However, analysis and comparison with "do-nothing" scenario in the morning peak period, increased the waiting time of individual vehicles at almost all of the analyzed intersections, i.e., the average waiting time at all analyzed intersections increased by $29.8 \%$, which is an expected consequence of the priority of tram vehicles. However, the conditional priority enabled the flow of private traffic, i.e., there was no blockade of traffic flow in the simulation. In the afternoon peak period, the average waiting time of private transport increased by $34.97 \%$.

Based on the analysis of the unconditional and conditional priority of tram vehicles, it can be concluded that the unconditional priority would lead to a complete disruption of individual traffic in the central part of the City of Zagreb, and therefore the travel time of trams would increase by $13.03 \%$. Conditional priority enables individual traffic and a reduction in tram travel time but increases the waiting times of individual vehicles by $32.38 \%$ on average in the morning and afternoon peak period in comparison to scenario "do-nothing". Figure 10 shows summed travel times on all tram lines in morning and afternoon peak periods in the existing state, "do-nothing" scenario, unconditional and conditional priority scenarios. Unconditional priority is the worst scenario with increase of travel time by $12.5 \%$ in comparison to the "do-nothing" scenario. As mentioned in Chapter 4.2.1. this is expected due to the blockade of the whole corridor. On the other hand, conditional priority achieves a reduction in tram travel times of $11.01 \%$ compared to the existing state, $14.56 \%$ compared to scenario "do-nothing," and $24.05 \%$ in comparison to unconditional priority. 


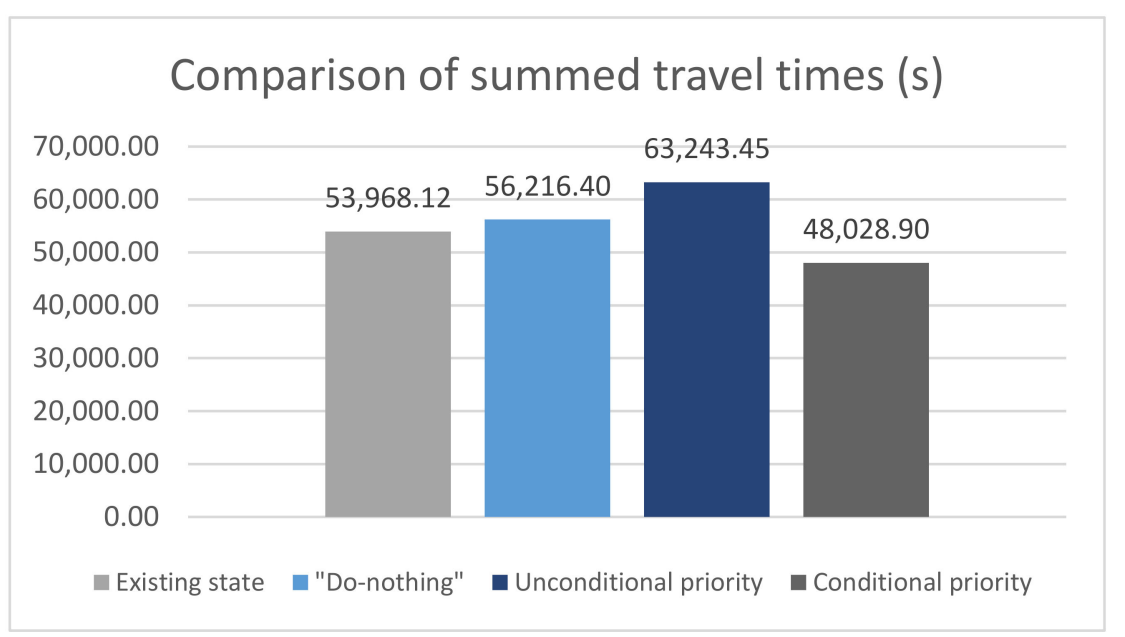

Figure 10. Comparison of summed travel time of all tram lines (s) in morning and afternoon peak periods.

\section{Discussion}

The aim of this study was to enable traffic flow in cases where PT is not physically separated from individual traffic and uses a common traffic lane. VISSIM, with its EPICS module, was used as a simulation tool. EPICS enables the assignment of weighting coefficients to individual signal groups, and direct implementation at real intersections is possible.

The basic problem addressed in this study was determination of the weight coefficients of the signal groups used by PT vehicles. The methodology was based on the counting of passengers in each of PT and individual vehicles. In further research, it is proposed to automate passenger counting and estimation. In the case of individual vehicles, cameras can be used at the intersections and, using video processing, the number of passengers in individual vehicles can be determined. For PT vehicles, video processing of recordings from cameras located in the vehicles themselves is proposed. Cameras placed at intersections can be also used to determine trapezoidal probability density function of PT arrival time to stop line.

Even though the EPICS module is user friendly, it should enable changing of several parameters and be more easily adapted to local conditions. In particular, it is especially necessary to work on its functioning at complex intersections where several signal groups contain PT lines. In addition, the methodology proposed in this paper addresses the gap that existed in the definition of the weight coefficient of signal groups.

In further research, it will be necessary to develop a methodology for the dynamic change in the weight factors, depending on the current ratio of the number of passengers at intersections.

In addition, the further development of EPICS should strive to allow it to work at a network level, rather than the local level in microsimulation tool. Even though BALANCE is intended for network optimization of signal plans, its development is extremely complex and first requires defining a macrosimulation model with origin-destination matrices in VISUM prior to exporting to VISSIM.

\section{Conclusions}

Compared to previous studies, a novelty is using the actual relationship between passenger flows in PT and individual vehicles and the adjustment of signal plans at each intersection depending on that relationship. Developing a methodology for determining the weight factors of particular signal groups at intersections has enabled individual traffic movement in conditions of PT priority. This methodology has proven successful on an extensive and congested network. Furthermore, the proposed methodology addresses the gap that existed in the definition of the weight coefficients of signal groups in EPICS. 
As a result of the research conducted in this study, it was possible to develop a methodology for the conditional priority of PT vehicles that simultaneously enables the flow of individual traffic, but with an increase in its waiting time. After the establishment of unconditional priority, there was a complete blockade of personal and public transport due to the high frequency of trams and frequent minimal green times on opposite directions. To enable movement of private and public transport, conditional priority, based on the weighting coefficients of conflicting passenger flows in personal vehicles and public transport, was approached. The results of conditional priority showed average reduction in travel time in the morning peak period from $7.64 \%$ to $18.76 \%$ and in the afternoon peak period, improvements ranged from $5.60 \%$ to $22.50 \%$, compared to "do nothing" scenario. These results are comparable to the results in other cities where EPICS was used. Still, they depend on the PT traffic management (mixed or dedicated lanes), road capacity, traffic volumes and frequency of PT lines.

The measurement of the effects of prioritizing PT vehicles must be undertaken in conjunction with a change in the city's transport policy, to encourage PT transport and discourage mass travel by car to the city center.

Author Contributions: Conceptualization, L.N. and K.B.; methodology, L.N. and L.D.; software, L.D. and K.B.; validation, L.N., K.B. and L.D.; formal analysis, T.R.; data curation, K.B.; writingoriginal draft preparation, L.N., L.D. and K.B.; writing-review and editing, L.N., L.D., K.B. and T.R.; visualization, T.R.; supervision, L.N. All authors have read and agreed to the published version of the manuscript.

Funding: This research was funded by the City of Zagreb, City Office for Physical Planning, Construction of the City, Utility Services and Transport.

Institutional Review Board Statement: Not applicable.

Informed Consent Statement: Not applicable.

Data Availability Statement: The data presented in this study are available on request from the corresponding authors. The data are not publicly available because certain measures are still need to be used in other studies.

Conflicts of Interest: The authors declare no conflict of interest.

\section{References}

1. Sunkari, S.R.; Beasley, P.S.; Urbanik, T.; Fambro, D.B. Model to evaluate the impacts of bus priority on signalized intersections. Transp. Res. Rec. 1995, 1494, 117-123.

2. Urbanik, T.; Holder, R.W. Evaluation of Priority Techniques for High Occupancy Vehicles on Arterial Streets; Texas Transportation Institute, Texas A \& M University: College Station, TX, USA, 1977.

3. Urbanik, T. Priority treatment of buses at traffic signals. Transp. Eng. 1997, 47, 31-33.

4. Kim, W.; Rilett, L.R. Improved Transit Signal Priority System for Networks with Nearside Bus Stops. Transp. Res. Rec. J. Transp. Res. Board 2005, 1925, 205-214. [CrossRef]

5. Smith, H.R.; Hemily, P.B.; Ivanovic, M. Transit Signal Priority (TSP): A Planning and Implementation Handbook; ITS America: Washington, DC, USA, 2005.

6. Taube, R.N. Bus Actuated Signal Preemption Systems: A Planning Methodology; Center for Urban Transportation Studies, University of Wisconsin-Milwaukee: Milwaukee, Wisconsin, 1976.

7. Held, B. Bus Priority: A Focus on the City of Melbourne; Department of Civil Engineering, Monash University: Clayton, VIC, Australia, 1991.

8. Liu, H.; Skabardonis, A.; Li, M. Simulation of Transit Signal Priority Using the NTCIP Architecture. J. Public Transp. 2006, 9, 117-136. [CrossRef]

9. Chandler, C.; Hoel, L.A. Effects of Light Rail Transit on Traffic Congestion; Center for Transportation Studies, University of Virginia: Charlottesville, VA, USA, 2004.

10. Nor Azlan, N.N.; Md Rohani, M. Overview of Application Of Traffic Simulation Model. Matec Web Conf. 2018, 150, 03006. [CrossRef]

11. Anderson, P.; Daganzo, C.F. Effect of Transit Signal Priority on Bus Service Reliability. Transp. Res. Procedia 2019, 38, 2-19. [CrossRef]

12. Ghaffari, A.; Mesbah, M.; Khodaii, A. Designing a transit priority network under variable demand. Transp. Lett. 2019, 12, 427-440. [CrossRef] 
13. Haitao, H.; Yang, K.; Liang, H.; Menendez, M.; Guler, S.I. Providing public transport priority in the perimeter of urban networks: A bimodal strategy. Transp. Res. Part C Emerg. Technol. 2019, 107, 171-192. [CrossRef]

14. Ji, Y.; Tang, Y.; Shen, Y.; Du, Y.; Wang, W. An Integrated Approach for Tram Prioritization in Signalized Corridors. IEEE Trans. Intell. Transp. Syst. 2020, 21, 2386-2395. [CrossRef]

15. Zhou, W.; Bai, Y.; Li, J.; Zhou, Y.; Li, T. Integrated Optimization of Tram Schedule and Signal Priority at Intersections to Minimize Person Delay. J. Adv. Transp. 2019, 2019, 4802967. [CrossRef]

16. De Keyser, O.; Hillewaere, M.; Audenaert, P.; Maenhout, B. Optimising the public transport priority at road intersections. Iet Intell. Transp. Syst. 2018, 12, 986-994. [CrossRef]

17. Zlatkovic, M.; Stevanovic, A.; Martin, P.T.; Tasic, I. Evaluation of Transit Signal Priority Options for Future Bus Rapid Transit Line in West Valley City, Utah. Transp. Res. Rec. J. Transp. Res. Board 2012, 2311, 176-185. [CrossRef]

18. Polgár, J.; Tettamanti, T.; Varga, I. Passenger number dependent traffic control in signalized intersections. Period. Polytech. Civ. Eng. 2013, 57, 201. [CrossRef]

19. Vujić, M.; Mandzuka, S.; Greguric, M. Pilot Implementation of Public Transport Priority in the City of Zagreb. Promet-Traffic Transp. 2015, 27, 257-265. [CrossRef]

20. Wahlstedt, J. Evaluation of the two self-optimising traffic signal systems Utopia/Spot and ImFlow, and comparison with existing signal control in Stockholm, Sweden. In Proceedings of the 16th International IEEE Conference on Intelligent Transportation Systems (ITSC 2013), The Hague, The Netherlands, 6-9 October 2013. [CrossRef]

21. Ezzat, A.A.; Farouk, H.A.; El-Kilany, K.S.; Moneim, A.F. Optimization using simulation of traffic light signal timings. In Proceedings of the 2014 Internacional Conference on Industrial Engineering and Operations Management Bali, Bali, Indonesia, 7-9 January 2014; pp. 1428-1433.

22. Kaczmarek, M.; Rychlewski, J. Tram priority traffic control on complex intersections. IFAC Proc. Vol. 2006, 39, 416-420. [CrossRef]

23. Ou, D.; Yan, H.; Li, H.; Li, W. Optimization of Conflicting Tram Signal Priority Requests Based on Spatiotemporal Interlocking Logic Using Microscopic Simulation. Int. J. Softw. Eng. Knowl. Eng. 2018, 28, 507-522. [CrossRef]

24. Stevanovic, J.; Stevanovic, A.; Martin, P.T.; Bauer, T. Stochastic optimization of traffic control and transit priority settings in VISSIM. Transp. Res. Part C Emerg. Technol. 2008, 16, 332-349. [CrossRef]

25. PTV AG. Krakow Focusses on Adaptive Control Systems by PTV Group-Success Story PTV Balance and PTV Epics. Available online: https://www.ptvgroup.com/fileadmin/user_upload/Products/PTV_Balance/Documents/Success_Stories/PTV_ Balance_SuccessStory_Krakow_EN.pdf (accessed on 30 April 2021).

26. PTV AG. PTV BALANCE User Manual; PTV: Karlsruhe, Germany, 2016.

27. PTV AG. PTV EPICS User Manual; PTV: Karlsruhe, Germany, 2019.

28. PTV AG. The Tristar and Kraków Systems a PTV Balance and PTV Epics Case Study. Available online: http://212.67.214.5 9/Symposium/Symposium2015/PapersForDownload/The\%20Tristar\%20\&\%20Krakow\%20system\%20-\%20a\%20PTV\%20 Balance\%20and\%20PTV\%20Epics\%20case\%20study.pdf (accessed on 30 April 2021).

29. Klanac, I.; Stevanovic, A.; Radivojevic, D.; Soltani-Sobh, A.; Ostojic, M. Evaluation of Epics Adaptive Traffic Signal Control in Microsimulation Environment. 95th Transportation Research Board Annual Meeting, Washington, DC, USA. 2016. Available online: https:/ /www.researchgate.net/publication/325115789_Evaluation_of_Epics_Adaptive_Traffic_Signal_Control_ in_Microsimulation_Environment (accessed on 10 April 2021).

30. Public Transport Operator of City of Zagreb. Available online: https:/ /www.zet.hr/en (accessed on 20 September 2019).

31. Mertz, J. Ein mikroskopisches Verfahren zur verkehrsadaptiven Knotenpunktsteuerung mit Vorrang des öffentlichen Verkehrs. Ph.D. Thesis, Technische Universität München, Munich, Germany, 2001.

32. NSW Government. Transport Roads and Maritime Services. Traffic Modelling Guidelines, version 1, London. 2013. Available online: https:/ / roads-waterways.transport.nsw.gov.au/business-industry/partners-suppliers /documents/technical-manuals / modellingguidelines.pdf (accessed on 15 March 2021).

33. Babojelić, K.; Novačko, L. Modelling of Driver and Pedestrian Behaviour-A Historical Review. Promet-Traffic Transp. 2020, 32, 727-745. [CrossRef]

34. TAG UNIT M3. 1: Highway Assignment Modelling; Department for Transport: London, UK, 2013.

35. Phase I of the Master Plan of the Transport System of the City of Zagreb, Zagreb County and Krapina-Zagorje County. City of Zagreb. 2018. Available online: http:/ /www.ipzp.hr/en/master-plan/ (accessed on 8 March 2021). 